\title{
Fiber transmission and generation of ultrawideband pulses by direct current modulation of semiconductor lasers and chirp-to-intensity conversion
}

\author{
Víctor Torres-Company, ${ }^{1}$ Kamau Prince, ${ }^{2}$ and Idelfonso Tafur Monroy ${ }^{2, *}$ \\ ${ }^{1}$ Departament de Física, GROC-UJI, Universitat Jaume I, 12071 Castelló, Spain \\ ${ }^{2}$ Department of Communications, Optics and Materials, Technical University of Denmark, \\ 2800 Kgs. Lyngby, Denmark \\ *Corresponding author: itm@com.dtu.dk
}

\author{
Received November 15, 2007; accepted December 15, 2007; \\ posted January 7, 2008 (Doc. ID 89697); published January 22, 2008
}

\begin{abstract}
Optical pulses generated by current modulation of semiconductor lasers are strongly frequency chirped. This effect has been considered pernicious for optical communications. We take advantage of this effect for the generation of ultrawideband microwave signals by using an optical filter to achieve chirp-to-intensity conversion. We also experimentally achieve propagation through a $20 \mathrm{~km}$ nonzero dispersion shifted fiber with no degradation of the signal at the receiver. Our method constitutes a prospective low-cost solution and offers integration capabilities with fiber-to-the-customer-premise systems. (C) 2008 Optical Society of America OCIS codes: $060.4510,070.0070,250.5960,350.4010$.
\end{abstract}

In the past few years, there has been a growing interest in the generation of ultrawideband (UWB) microwave signals. The UWB radio-frequency $(\mathrm{RF})$ spectrum is regulated between the 3.1 and $10.6 \mathrm{GHz}$ band with a power spectral density of $-41.3 \mathrm{dBm} / \mathrm{MHz}$ [1]. Due to the high-data-rate capabilities, low power consumption, and immunity to multipath fading, these signals have an unprecedented opportunity to impact radio communication systems and promise as well substantial applications in radar, safety, and biomedicine. Although a large number of electronic devices for providing UWB pulses exist, optical technologies offer a viable alternative with several advantageous features. With optical approaches, the achieved bandwidth is usually limited only by the optoelectronic $(\mathrm{O} / \mathrm{E})$ conversion bandwidth. More important, all-optical UWB signal generation schemes constitute an ideal scenario for seamless integration of the distribution of wireless communications signals over optical fiber links.

Optical schemes to generate UWB signals include the use of a Fourier-transform geometry for shaping the power spectrum of an ultrashort light pulse [2-4]. The UWB signal is achieved by stretching the pulse in a fiber and by subsequent photodetection. In this way, a frequency-to-time mapping takes place. Later, an all-fiber variation was verified, achieving the generation of monocycle and doublet pulses only [5]. The same group also obtained UWB pulses by synthesizing a bandpass coherent microwave filter by combining an electro-optic phase modulator (EOPM) with fiber dispersion [6], acting globally as an electronic differentiator at low frequencies but implemented in the optical domain. More recently, some reported optical UWB signal generation approaches take advantage of the nonlinear optical properties of semiconductor optical amplifiers (SOAs) [7-9]. By exploiting cross-gain modulation [7], the output light of the
SOA consisted of two reversed polarized pulses. By further adjusting the time delay between the two pulses with a pair of fiber Bragg gratings, monocycle pulses were achieved. This pulse waveform has also been generated based on the intensity overshooting effect [9] of optical dark pulses amplified by an SOA with unsaturated optical gain. Cross-phase modulation in an SOA, combined with an optical filter to provide chirp-to-intensity conversion, has proved to be an alternative way to generate UWB monocycles [8]. A similar chirp-to-intensity conversion approach was demonstrated with an EOPM combined with a fiber Bragg-grating filter [10].

In this Letter, we propose an efficient method to generate UWB pulses based on chirp-to-intensity conversion requiring a single optical source. Our experimental setup consists of a distributed feedback (DFB) laser whose driving current is modulated by the electrical data signal. When the laser is biased far from the threshold, the generated pulses are strongly frequency chirped. For a long time, this effect has been considered as pernicious for pulse propagation in optical fibers due to chromaticdispersion issues [11]. However, by placing a spectral filter after the output of the DFB laser, a chirp-tointensity conversion takes place. The resultant intensity waveform after photodetection has an RF spectrum that meets the regulations for being considered as UWB. With respect to previous reported approaches, our configuration offers an efficient solution in terms of power consumption, since there are no optical nonlinear processes involved requiring additional light sources or additional active optical devices. Our approach requires a single optical light source and avoids the use of external electro-optic modulation, so low complexity is inherent to this generation scheme. We have further verified the capability of the new technique for a fiber-to-the-home sce- 
nario by propagating the signal through a $20 \mathrm{~km}$ nonzero dispersion shifted fiber (NZDSF). It is worth mentioning that a previous reported work [12] used a Fabry-Perot laser, current modulated for the generation of UWB signals. However, the frequency chirp was not used for achieving UWB signal generation. Instead, monocycle pulses were achieved by $\mathrm{O} / \mathrm{E}$ conversion and subsequent differentiation of Gaussian intensity pulses in the electrical domain.

Our experimental setup is schematically depicted in Fig. 1. A DFB laser (NEL NLK5C5EBKA) with a central wavelength of $1550.10 \mathrm{~nm}$ is used as a light source. The bias current is fixed to $-66.35 \mathrm{~mA}$. A $12.5 \mathrm{Gbit} / \mathrm{s}$ electrical signal from a pulse pattern generator (Anritsu MP17633) with a peak-to-peak voltage of $0.250 \mathrm{~V}$ is used for direct current modulation. Figure 2 depicts the temporal pulse laser shape measured with a $20 \mathrm{GHz}$ bandwidth photodiode at point A in Fig. 1. Because we are operating far from the threshold bias current the contribution of the frequency modulation is dominant versus the intensity modulation contribution. We profitted this chirp to achieve a monocycle pulse by placing a tunable optical bandpass filter (OBPF) with a Gaussian shaped transfer function at the output of the laser. Figure 3 shows the measured optical power spectrum at the output of the laser (solid curve), and the dasheddotted curve represents the measured optical filter transfer function. The measurements have been taken with an optical spectrum analyzer (Ando OSA) with a resolution bandwidth of $0.01 \mathrm{~nm}$. The plot represents the optimal placement of the OBPF, that is, when the laser spectrum lies at the linear slope of the OBPF transfer function. The average optical power level before the OBPF was measured to be 2.6 and $-4.0 \mathrm{dBm}$ at the input of the fiber, respectively.

Mathematically, frequency-chirp-to-intensity conversion can be denoted as follows. We can assume that the complex field of the frequency-chirped pulse is filtered by a linear optical filter $H(\omega)=A(\omega+\Delta \omega)$, where $A$ is a real constant that determines the slope of the filter and $\Delta \omega$ denotes the vertical offset from the zero-crossing point. The temporal envelope at the output of the filter is given by

$$
e_{\text {out }}(t)=j A\left[e_{\mathrm{in}}^{\prime}(t)-j \Delta \omega e_{\mathrm{in}}(t)\right],
$$

where the prime denotes temporal derivation and $e_{\text {in }}(t)$ is the input temporal envelope. We have taken

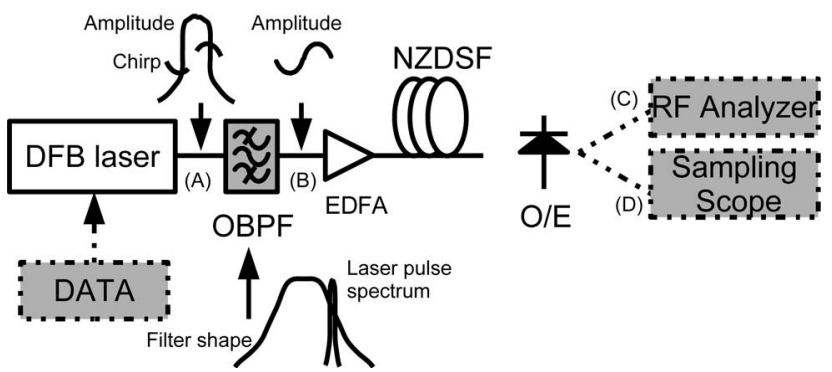

Fig. 1. Experimental setup. DFB, distributed feedback laser; OBPF, optical bandpass filter; O/E, optoelectronic conversion. NZDSF, nonzero dispersion shifted fiber; EDFA, erbium-doped fiber amplifier. Dotted lines, electrical connections.

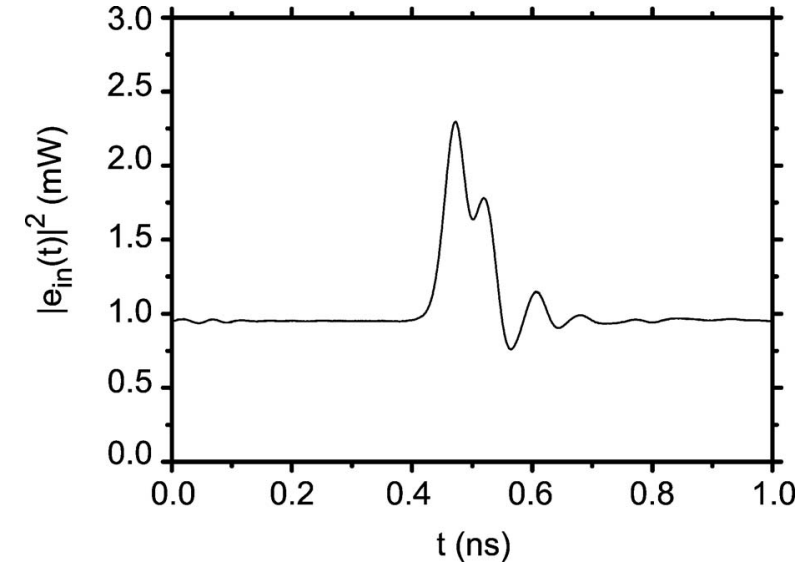

Fig. 2. Intensity pulse shape before the filter.

into account that $F T^{-1}\left[-j \omega E_{\text {in }}(\omega)\right]=e_{\text {in }}^{\prime}(t)$, where $E_{\text {in }}(\omega)=F T\left[e_{\text {in }}(t)\right]$, with $F T$ and $F T^{-1}$ denoting Fourier and inverse Fourier transformation, respectively. We can always rewrite the input envelope as $e_{\text {in }}(t)$ $=I_{o}^{1 / 2}(t) \exp [-j \Phi(t)]$, where $\Phi(t)$ and $I_{o}(t)$ denote the phase and intensity modulations, respectively. We now assume that the frequency modulation dominates with respect to the intensity modulation, but its contribution value is less than the corresponding frequency offset of the filter, i.e., $\left|I_{o}^{\prime}(t)\right| \ll\left|\Phi^{\prime}(t)\right| \ll \Delta \omega$. With this assumption, and after some straightforward algebra, the optical intensity at the output of the filter can be approximated to

$$
I_{\text {out }}(t) \approx 2 A^{2} \Delta \omega I_{o}(t)\left[\Phi^{\prime}(t)+\Delta \omega / 2\right] .
$$

The above equation establishes that the light intensity at the output of the optical filter is essentially given by the frequency chirp of the input optical pulse. The derivation of Eq. (2) follows the approach in $[8,10]$. Note that Eq. (2) includes the contribution of both chirp and intensity modulations. However, since the laser is biased far from the threshold, the frequency modulation dominates over the intensity, and it is expected that the resultant intensity waveform after spectral linear filtering comes from the chirp.

At point B in Fig. 1, the optical signal is amplified by an erbium-doped fiber amplifier (EDFA) and

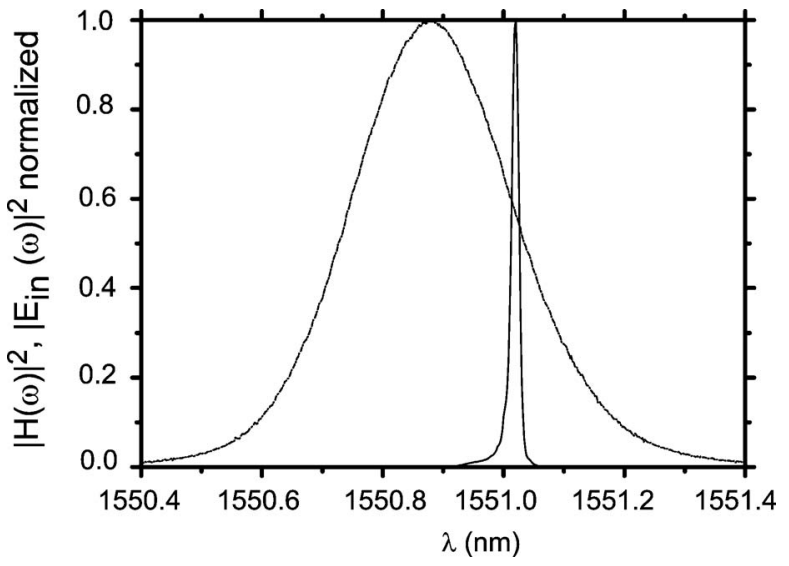

Fig. 3. Power spectrum of laser (solid curve) and OBPF (dashed-dotted curve). 
propagated through $20 \mathrm{~km}$ of a NZDSF with a $4.5 \mathrm{~dB}$ loss and a $5 \mathrm{ps} / \mathrm{nm} / \mathrm{km}$ dispersion. The intensity waveform at the output is $\mathrm{O} / \mathrm{E}$ converted by a $10 \mathrm{GHz}$ $3 \mathrm{~dB}$ bandwidth photodiode, which intrinsically assists in smoothing undesired high-frequency RF components that do not fall into the UWB range. The resultant electrical signal is measured in the $R F$ domain by an electrical spectrum analyzer (Agilent E4407B9) with a resolution of $1 \mathrm{MHz}$, and in the time domain by a sampling oscilloscope (Agilent Infinium 86100A). The EDFA is adjusted so that the receiver gets an average optical input power of $-0.5 \mathrm{dBm}$. Figure 4 shows the measured resultant UWB signal consisting of a monocyclelike pulse in time and frequency domains. For the frequency domain, we selected a repetition rate of $390.6 \mathrm{MHz}$ (a " 1 " followed by 32 " 0 ") so that the achieved spectral shape is properly sampled. As can be appreciated, the RF spectrum spreads over the UWB region. Due to the monocyclelike waveform, a nondisregarding low-frequency content still remains, which can be minimized by reducing the power into the photodiode with some extra attenuator.

In summary, a novel optical approach for the generation of UWB signals has been proposed and ex-
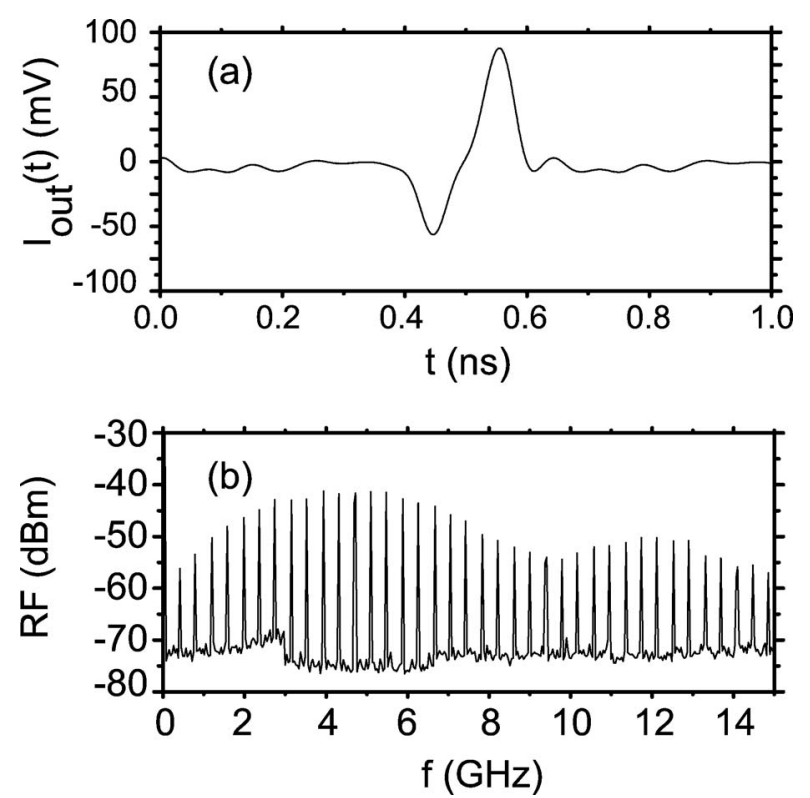

Fig. 4. UWB pulse achieved at the receiver: (a) intensity waveform and (b) RF spectrum. perimentally verified. It takes advantage of the frequency chirp of optical pulses generated by direct current modulation of semiconductor lasers. Essentially, chirp-to-intensity conversion is achieved by optical linear filtering of frequency-chirped optical pulses. We achieve a monocycle waveform that meets the UWB requirements. The proposed scheme is simple and compact since it avoids the use of any external electro-optic modulator and achieves high efficiency in terms of power consumption because nonlinear optical processes are involved.

This work has been partly funded by the Dirección General de Investigación Cientifica y Técnica, Spain and Fondos Europeos para el DEsarrollo Regional, under the projects FIS2007-62217 and SAUUL (Science and Applications of ultrafast ultraintense lasers) in the programme CONSOLIDER. V. Torres acknowledges financial support from a Formación de Profesorado Universitario grant from the Ministerio de Eduación y Ciencia. K. Prince acknowledges the Danish Agency for Science, Technology and Innovation International PhD Scholarship.

\section{References}

1. M.-G. Di Benedetto, T. Kaiser, A. F. Molish, I. Oppermann, and C. Politano, eds., UWB Communication Systems: A Comprehensive Overview (Hindawi, 2006).

2. J. Chou, Y. Han, and B. Jalali, IEEE Photon. Technol. Lett. 15, 581 (2003).

3. I. S. Lin, J. D. McKinney, and A. M. Weiner, IEEE Microw. Wirel. Compon. Lett. 15, 226 (2005).

4. J. D. McKinney, I. S. Lin, and A. M. Weiner, IEEE Trans. Microwave Theory Tech. 54, 4247 (2006).

5. C. Wang, F. Zeng, and J. P. Yao, IEEE Photon. Technol. Lett. 19, 137 (2007).

6. F. Zeng and J. P. Yao, IEEE Photon. Technol. Lett. 18, 823 (2006).

7. Q. Wang, F. Zeng, S. Blais, and J. P. Yao, Opt. Lett. 31, 3083 (2006).

8. J. J. Dong, X. L. Zhang, J. Xu, D. X. Huang, S. N. Fu, and P. Shum, Opt. Lett. 32, 1223 (2007).

9. J. J. Dong, X. L. Zhang, J. Xu, and D. X. Huang, Opt. Lett. 32, 2158 (2007).

10. F. Zeng and J. P. Yao, IEEE Photon. Technol. Lett. 18, 2062 (2006).

11. G. P. Agrawal, Fiber-Optic Communication Systems (Wiley Interscience, 2002).

12. W. P. Lin and Y.-C. Chen, IEEE J. Sel. Top. Quantum Electron. 12, 882 (2006). 